\title{
A Simplified Integration of Multi-Channel Ultrasonic Guided Wave System for Phased Array Detection and Total Focusing Imaging
}

\author{
Yan Lyu, Huaxing Hong, Guorong Song and Cunfu He \\ Faculty of Materials and Manufacturing, Beijing University of Technology, Beijing 100124, China. \\ E-mail: 1vyan@bjut.edu.cn.
}

\begin{abstract}
(Received 12 March 2020; accepted 29 August 2020)
Ultrasonic guided waves based on sensors array effectively improve the detection sensitivity of defects and realize the intuitive imaging of defects. In this research, a 16-channel guided wave excitation/acquisition system is simply integrated for the array focusing detection. Using a FPGA (Field Programmable Gate Array) as the main control unit, a high-voltage excitation of a multi-channel tone-burst signal with synchronous signal acquisition is designed. Experiments are conducted by the developed multi-channel system and the piezoelectric linear array. A guided wave phased array and the total focusing imaging algorithm are demonstrated on a $1 \mathrm{~mm}$ aluminum plate. The experimental results show that the system can meet the practical requirements of guided wave array focusing detection. By combining the total focusing method and the phase-based sign coherence factor, the compounded image shows that the artifacts can effectively be reduced, and the contrast is improved. Meanwhile, this system can solve the problem of distributed sensor array detection, in which the process is cumbersome and inefficient previously. Through the system and the sensor array, the guided wave phased focusing method and the composite total focusing method can effectively improve the sensitivity and the positioning accuracy of defect detection in thin plate.
\end{abstract}

\section{INTRODUCTION}

Non-destructive evaluation based on guided waves can improve the detection range, ${ }^{1-4}$ and the sensor array can overcome the low resolution and energy dispersion for single sensor. Through a variety of array forms, large-scale and highprecision detection of defects will be achieved, with the characteristics of guided waves. ${ }^{5,6}$ The total focusing imaging method based on sensor array can detect and locate multiple defects simultaneously. Also, it solves the problem of identification of small defects that smaller than a half wavelength. ${ }^{7,8}$ A phased array system controls every element in the array to excite and receive ultrasonic waves according to a certain time delay. The beam can be focused in any point of a testing structure, which can improve the capability of detecting small defects. $^{9-12}$ However, currently commercial multi-channel guided wave phased array systems are far more expensive, and the hardware and software have been highly integrated, which is difficult for re-development for specific experiments. ${ }^{13,14}$ Therefore, a multi-channel experimental guided wave focusing system is established in this research to facilitate phased array measurements and total focusing non-destructive detection. For plate-type specimens, the array focusing detection and total focusing imaging of defects on an aluminum plate have been achieved, starting from multi-channel system design to guided wave experiments.

For the ultrasound array total focusing method, Holmes ${ }^{15}$ firstly proposed a full matrix capture (FMC) model and a total focusing method (TFM) based on the full matrix acquisition model. The matrix array controller (MAC) and a digital storage oscilloscope (DSO) were used for signal excitation and acquisition. Each experiment needed to collect 2080 groups of experimental signals, which took a long time. Jeune $^{16}$ filtered out the signals for imaging by setting a threshold, which enabled adaptive fast total focusing imaging of the weld transverse holes. Also, the experiment used a commercial portable system, Gekko (manufactured by M2M), which can realize TFM imaging in real time. $\mathrm{Li}^{17}$ used a commercial array controller Micropulse 4A (manufactured by Peak NDT Ltd.) and applied the improved TFM algorithm to defect detection of composite materials. But these commercial devices are difficult to perform distributed sensor array excitation.

Aiming at the problem that TFM only uses the amplitude information of the detection signals, which makes the imaging affected by noise, side lobes and grating lobes, many researchers pay attention to another important information of the detection signal - "phase". ${ }^{18,19}$ Then, a phase imaging algorithm, which can suppress side lobes and grating lobes and improve the signal-to-noise ratio, was developed. $\mathrm{Liu}^{20}$ used a dense rectangular piezoelectric transducer array, combined with amplitude and phase, to achieve the multi-defect detection based on composite imaging. The experiments used a waveform generator (Tektronix AFG3021B) for excitation and a digital oscilloscope (Tektronix DPO 4054) for acquisition. Thus, the system can only be used for single-channel excitation. Frequent switching between excitation and receiving elements are required for full matrix data acquisition, which is tedious and inefficient.

In this research, a 16-channel guided wave focusing detection system was developed, which can simultaneously excite and acquire signals from multiple sensors. Combined with a controlling program, the system can automatically achieve full matrix data collection. Above all, the system is simple, efficient, stable, and low cost. The research mainly contains three parts: design scheme of multi-channel guided wave detection system, phase focusing detection and amplitude total focusing imaging. Experiments were carried out on a $1 \mathrm{~mm}$ aluminum plate to verify the function and practicability of the system. The optimization of the imaging results of the thin plate by 


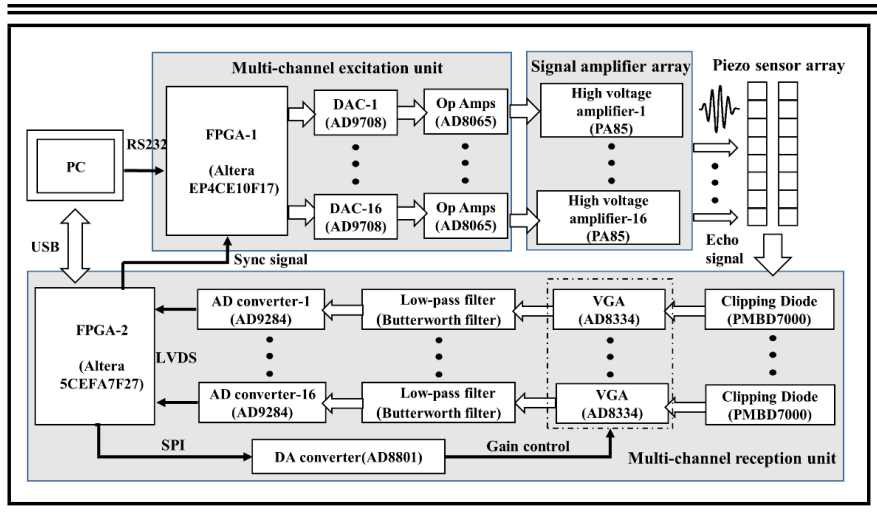

Figure 1. Framework of the System.

Table 1. System Parameters.

\begin{tabular}{|c|c|}
\hline Number of channels & 16 \\
\hline Excitation frequency & $10 \mathrm{kHz} \sim 1 \mathrm{MHz}$ \\
\hline Excitation amplitude & $150 \mathrm{Vpp}$ \\
\hline Excitation type & tone-burst signal \\
\hline Excitation delay resolution & $5 \mathrm{~ns}$ \\
\hline Programmable gain amplifier & $0 \sim 42 \mathrm{~dB}$ \\
\hline Sampling rate \& resolution & $50 \mathrm{MS} / \mathrm{s} \& 8 \mathrm{Bit}$ \\
\hline
\end{tabular}

guided wave total focusing algorithm will be implemented as well. Based on the combination of phase-based sign coherence factor imaging and the amplitude total focusing imaging, the contrast of defect imaging can be improved, while the artifact effect caused by noise can also be reduced.

\section{SYSTEM DESIGN}

\subsection{Overall Structure}

The multi-channel guided wave detection system consisted of a 16-channel signal generator, a high voltage amplifier array, a 16-channel synchronous acquisition board, a data transmission interface based on USB, a power module, and a computer. The overall design block diagram of the system is shown in Fig. 1. The multi-channel excitation and acquisition system was controlled by a FPGA (Field Programmable Gate Array). The host computer transmitted the parameters of the excitation system to the excitation main control FPGA through the RS232 serial port to generate 16-channel guided wave excitation signals. The excitation signals were then boosted by the respective amplifier circuits to excite the piezoelectric sensor array. The reflected echo signals received by each sensor were collected by high-speed ADC (Analog to Digital Converter) after passing through the clipping circuit and the signal conditioning circuit, the data was transmitted to the host computer through USB. The specifications of the system are shown in Table 1.

\subsection{Multi-Channel Signal Excitation}

The multi-channel guided-wave excitation module was based on the FPGA and DAC (Digital to Analog Converter). It realized the generation of 16 channels of arbitrary waveforms through DDS (Direct Digital Synthesis) technology. Usually, to reduce the dispersion of the guided wave and focus the energy on the main lobe, a sinusoidal signal modulated by a Hanning window is generated for guided wave propagation. The multi-channel excitation module required that multiple channels can transmit the excitation at the same time or accurately perform time delay controlled by a phase control rule. So, the controller needed to provide an accurate timing sequence.

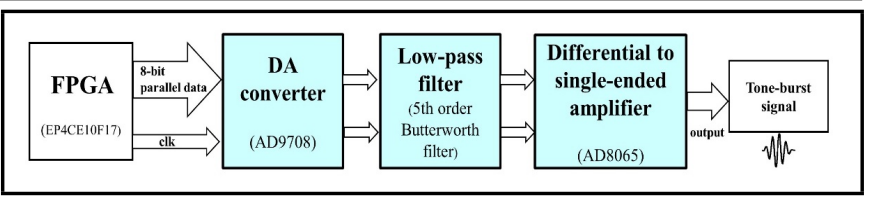

Figure 2. Single channel excitation scheme.

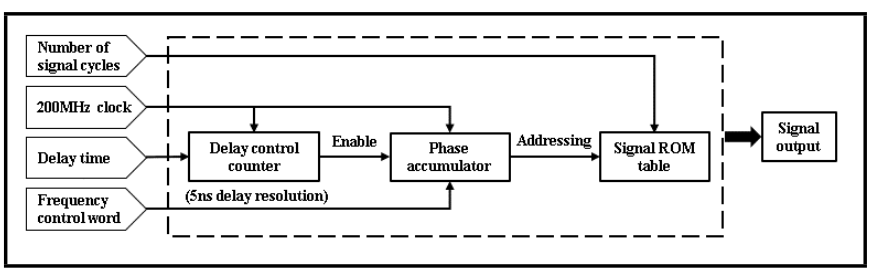

Figure 3. Waveform control block diagram.

The signal generation module consisted of a parallel DAC, a 5th order low-pass Butterworth filter, and an operational amplifier by AD8065. The clock pin and data pin of the DAC were connected to the FPGA, and the output data of the FPGA was converted into an analog signal. The differential output of the DAC was connected to the 5th order low-pass Butterworth filter, and the output of the filter is connected to AD8065, which converts the differential current output of the DAC into a single-ended voltage output, serving as the signal amplification output of the first stage. The single-channel signal generation scheme is shown in Fig. 2, which is the same for the other channels.

The waveform signal generation method was based on DDS technology, which was built in the FPGA. Waveform data was stored in the ROM of the FPGA as an amplitude reference table. Building a 32-bit phase accumulator. The accumulation amount of the phase accumulator at each clock was determined by the frequency control word, which can modulate the accumulation step to change the waveform frequency. Meanwhile the phase accumulator outputs the corresponding waveform amplitude by addressing in the ROM. The output frequency of DDS was:

$$
f_{\text {out }}=\frac{f_{c l k}}{2^{N}} \times M
$$

where $f_{c l k}$ was the system clock frequency, $N$ was the number of phase accumulator bits, and $M$ was the frequency control word.

To achieve the phased focusing, each channel should be able of delay excitation. Therefore, a delay control counter was set up. When the count reached the delay time of the external input, the DDS module was enabled, and the phase accumulator started to count. Through the PLL (Phase Locked Loop), the main frequency of the FPGA was doubled to $200 \mathrm{MHz}$ as the driving clock, which drives the delay control counter and the phase accumulator. So, the delay resolution was $5 \mathrm{~ns}$. The block diagram of waveform generation control is shown in Fig. 3.

\subsection{Signal Amplifier}

To generate a high-energy excitation signal and drive a piezoelectric sensor, a PA85 high-voltage power amplifier chip was used as the core to design a negative feedback signal amplification. Its dual power supply voltage was $\pm 100 \mathrm{~V}$, the voltage conversion rate was $800 \mathrm{~V} / \mu \mathrm{s}$, according to the configuration, with a 100 times magnification. The power bandwidth was $500 \mathrm{kHz}$, and the amplitude reached up to $150 \mathrm{Vpp}$ 


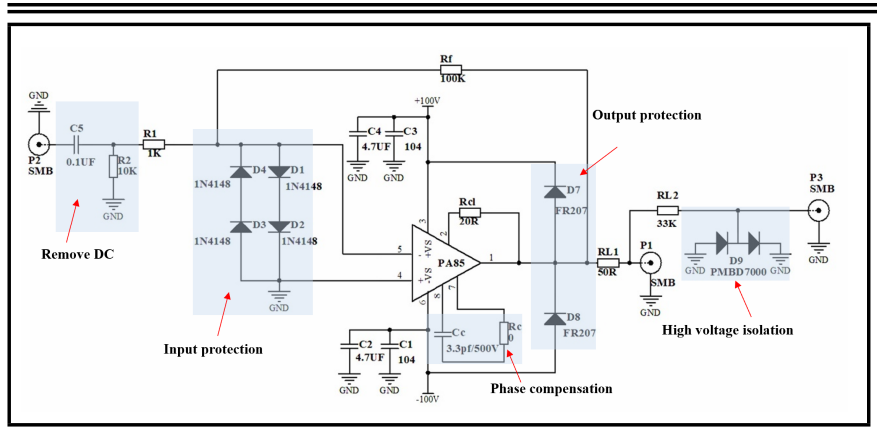

Figure 4. High voltage power amplifier circuit diagram.

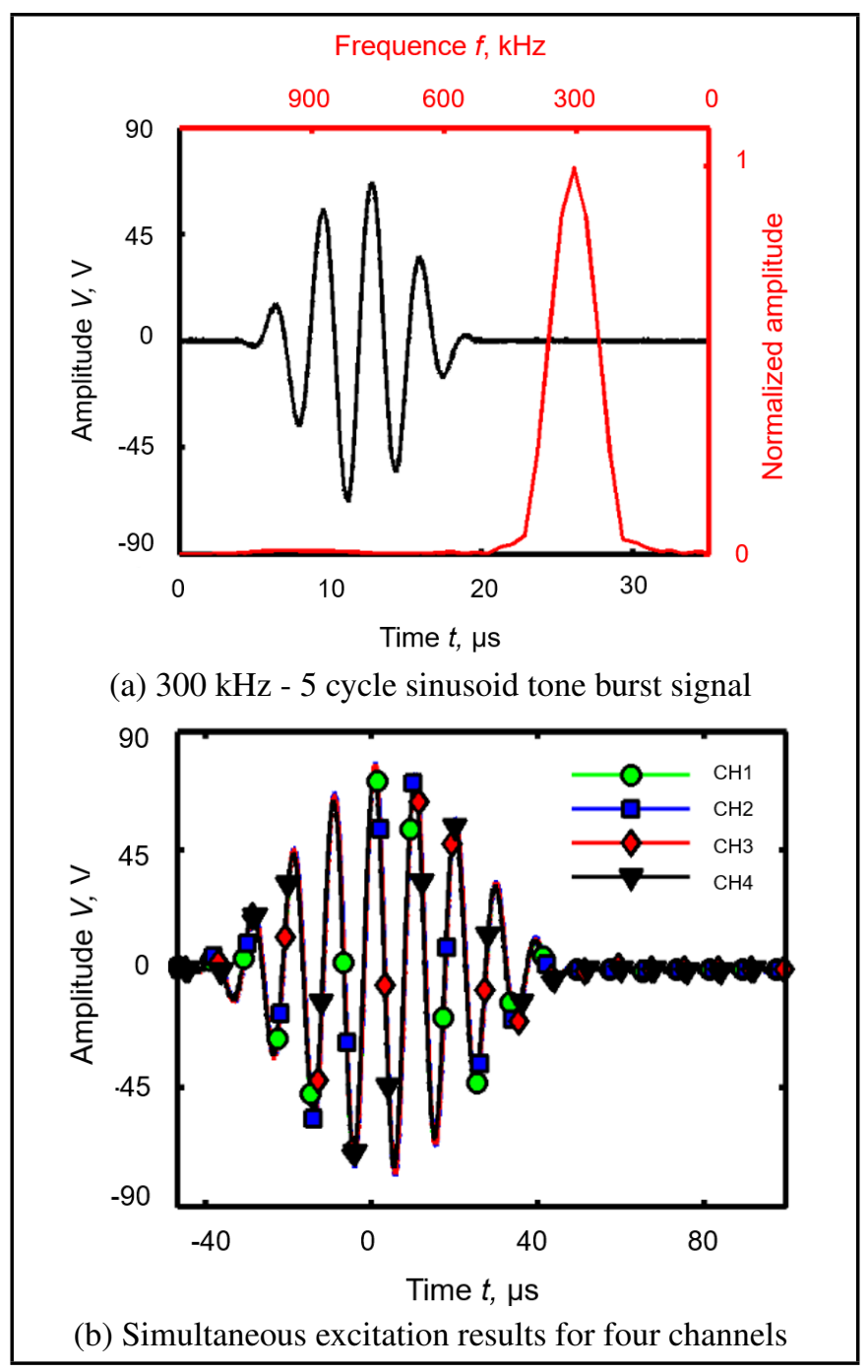

Figure 5. Excitation signal test.

when the output signal frequency was below $500 \mathrm{kHz}$. The 16-channel signal excitation system includes 16 high-voltage power amplifier modules. The power amplifier circuit design is shown in Fig. 4.

R2 and C5 removed the DC component of the input signal. Four diodes D1, D2, D3 and D4 (IN4148) were used to clamp the input signal amplitude at $1.5 \mathrm{~V}$ to protect the input. Rcl was the current limiting resistor. Rc and $\mathrm{Cc}$ were phase compensation resistors and capacitors to ensure the stability of the amplifier's gain bandwidth. D7 and D8 were output protection diodes. The power supply was designed with a transformer rectifier filter circuit, which converted the $220 \mathrm{~V}$ input to a $\pm 100 \mathrm{~V}$ DC power supply. The output voltage ripple of this

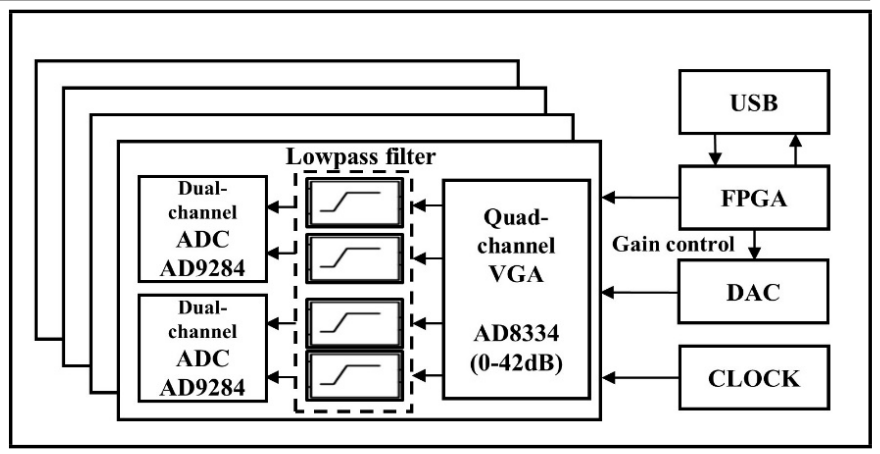

Figure 6. Multi-channel acquisition system structure.

linear power supply seemed to be low, which was conducive to improving the signal-to-noise ratio of the echo signal. The experimental result of the output excitation signal is shown in Fig. 5.

Figure 5(a) shows the time/frequency domain of the 5-cycle tone burst signal with a frequency of $300 \mathrm{kHz}$. It can be seen from the time domain that the peak value of the excitation signal reached up to $150 \mathrm{Vpp}$ without waveform distortion, the signal-to-noise ratio reached $38 \mathrm{~dB}$. The testing results of the simultaneous excitations of four are shown in Fig. 5(b). The signals of each channel coincided with each other very well, which indicates that the channels possess good consistency.

\subsection{Synchronous Signal Acquisition}

The 16-channel synchronous acquisition module consisted of a signal clipping circuit, a signal conditioning circuit, an ADC, and a main control FPGA. The signal received by each channel needed to be processed by program-controlled gain, filtering, etc. Then, it was converted to digital signals by a high-speed ADC and transmitted to the computer by FPGA. The overall scheme of the multi-channel synchronous acquisition module is shown in Fig. 6.

Taking the cost and integration into consideration, one quadchannel VGA (Voltage-controlled Gain Amplifier, AD8334) and two dual-channel AD converters (AD9284, 50MS/s sampling rate) simply constituted a module to achieve the acquisition and amplification of 4 input signals. Then, 4 such modules handled the simultaneous acquisition of 16 input signals. The 8 external sampling clocks required by the AD9284 were fanned out by an 8-channel clock (65LVDS108). Finally, the $50 \mathrm{MHz}$ sampling clock source crystal was fanned out to provide clock signals for 8 ADCs. Every two channels in AD8334 shared one gain control voltage. Then, totally 8 gain control voltage inputs needed to be provided. Therefore, an 8-channel, 8-bit $\mathrm{D} / \mathrm{A}$ converter (AD8801) was used to implement the gain control function.

The data transmission between the acquisition module and the host computer adopted USB communication to realize the bidirectional transmission of data and control instructions. It should be mentioned that the multi-channel signal acquisition computer program was based on CH376's dynamic link library, which automatically realized the full matrix data acquisition and storage.

\section{PRINCIPLE OF IMAGING}

\subsection{TFM Imaging}

TFM is a virtual focusing post-processing imaging technology based on full matrix data. To perform the full matrix data 
acquisition, every channel was sequentially excited, and then all the sensors received echoes simultaneously for each excitation. If the transducer array contained $N$ elements. Totally $N \times N$ sets of echo signals constructed the full matrix data.

The total focusing algorithm discretized the target area into many grids at the beginning. Then, it performed a virtual superposition of the signals of all the excitation sensors and receiving sensors in the array on the discrete grid points $(x, y):{ }^{15}$

$$
I(x, y)=\sum_{i=1}^{N} \sum_{j=1}^{N} P_{i j}\left[t_{i j}(x, y)\right]
$$

where $N$ was the number of the piezo-elements, which was 8 in this research, and $t_{i j}(x, y)$ represented the travelling time between the transmitting element $i$ to the focal point and returning back to the receiving element $j$ at detection point $(x, y)$, and $P_{i j}$ represented the amplitude of the ultrasonic signal corresponding to the transducer element $i$ and $j$ at point $(x, y)$.

\subsection{SCF Imaging}

In practical applications, total focusing imaging induces energy inconsistency by the directivity of the array element. Also, the different sound paths will cause different diffusion attenuation. In addition, one signal noise may casually produce false defects. Then, the phase coherent imaging algorithm, using the phase information of signals, is introduced. It can effectively suppress the gratings and side lobes of the sound beam and improve the resolution of the imaging result. Among the phase coherent imaging methods, the sign coherence factor (SCF) algorithm works relatively better in defect localization. $^{21}$ This method is based on the analysis of phase sign variance in $P_{i j}$.

Firstly, get the sign polarity $b_{i j}(x, y)$ of all signals in an matrix, which was expressed as:

$$
b_{i j}(x, y)=\left\{\begin{array}{l}
+1, P_{i j}\left[t_{i j}(x, y)\right] \geq 0 \\
-1, P_{i j}\left[t_{i j}(x, y)\right]<0
\end{array}\right.
$$

Then, using the polar information, we defined the sign coherence factor imaging matrix as:

$$
I_{S C F}(x, y)=1-\sigma=1-\sqrt{1-\left(\frac{1}{N^{2}} \sum_{i=1}^{N} \sum_{j=1}^{N} b_{i j}(x, y)\right)^{2}}
$$

where $\sigma$ was the standard deviation of the sign polarity $b_{i j}(x, y)$. To further suppress the side lobe effect, a modulation factor $s$ was added to the SCF imaging:

$$
I_{S C F}^{S}(x, y)=1-\left[\sqrt{1-\left(\frac{1}{N^{2}} \sum_{i=1}^{N} \sum_{j=1}^{N} b_{i j}(x, y)\right)^{2}}\right]^{s} ;
$$

where $s \geq 0$. The larger the $s$ approaches, the lower the side lobe effect was, and meanwhile it was lower than the main lobe at the same time. In this research, we used a typical value of $s=1$. For SCF imaging, each imaging pixel was related to the summation of the algebraic symbols in the sign matrix. At the defective locations, the sign coherence factor showed a

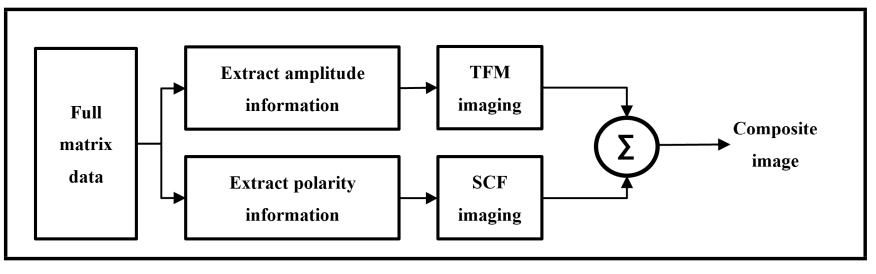

Figure 7. Composite imaging principle.

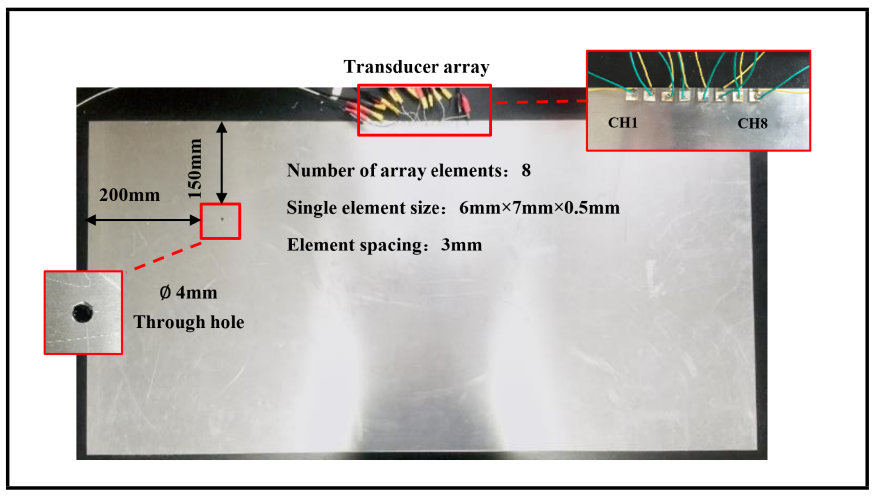

Figure 8. Phased array focusing experiment.

Table 2. Material parameters of the aluminum plate.

\begin{tabular}{|c|c|c|}
\hline Density & Poisson's ratio & Elastic Modulus \\
\hline $2700 \mathrm{~kg} / \mathrm{m}^{3}$ & 0.33 & $70 \mathrm{GPa}$ \\
\hline
\end{tabular}

relatively large value. If in the non-defective area, the algebraic symbols were random. Then, the summation approached zero. $^{22}$ Based on this phenomenon; defects were located by using SCF imaging.

\subsection{Composite Imaging}

In addition, by combining the resolution of TFM and the contrast of SCF, we located the defect more accurately. The main principle of this imaging method is shown in Fig. 7. In this research, the composite image was a superposition of the imaging results from TFM and SCF with weightings of $1: 1$.

\section{IMPLEMENTATIONS}

\subsection{Guided Wave Phased Array Focusing}

Actually, in order to verify the multi-channel guided wave detection system and to evaluate its focusing performance, a beam focusing experiment was performed on a thin aluminum plate, with a size of $1000 \mathrm{~mm} \times 500 \mathrm{~mm} \times 1 \mathrm{~mm}$. The material parameters are shown in Table 2 . The piezoelectric transducer was of length telescopic type with a size of $6 \mathrm{~mm} \times 7 \mathrm{~mm}$ $\times 0.5 \mathrm{~mm}$. The geometrical arrangement of the piezoelectric array and the artificial defects on the aluminum plate are shown in Fig. 8.

The dispersion curves of the $1 \mathrm{~mm}$ aluminum plate were calculated from Disperse (Imperial College, UK) according to the material parameters in Table 2, as shown in Fig. 9. The $S_{0}$ mode, with almost no dispersion in the low frequency range and fastest wave speed, will be easy to distinguish in the time domain waveform. Therefore, the $S_{0}$ mode was selected for the detection. The excitation signal was a 5-cycle sinusoid tone burst signal centered at $325 \mathrm{kHz}$. The central distance of the array element was generally half a wavelength, ${ }^{12}$ which was $9 \mathrm{~mm}$. Since the element width was $6 \mathrm{~mm}$, the array element spacing will be $3 \mathrm{~mm}$. 


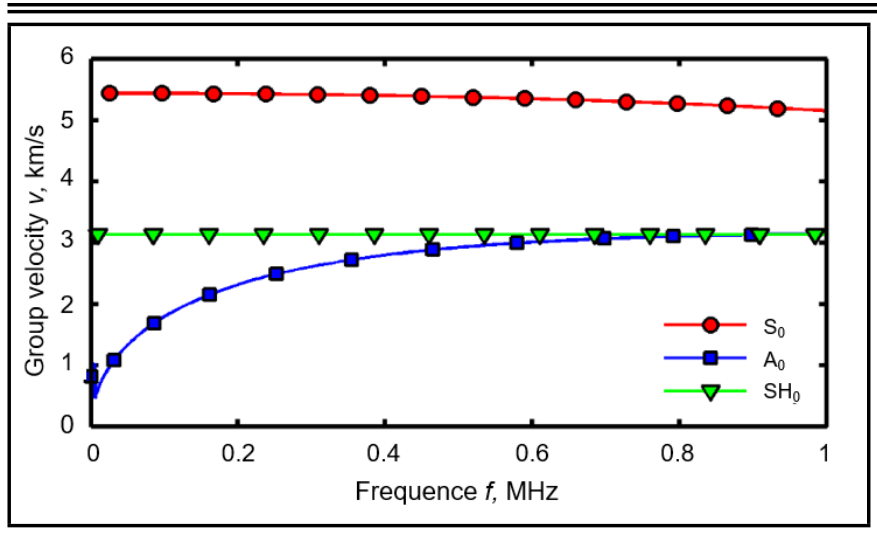

Figure 9. Dispersion curves of lamb waves in a $1 \mathrm{~mm}$-thick aluminum plate.

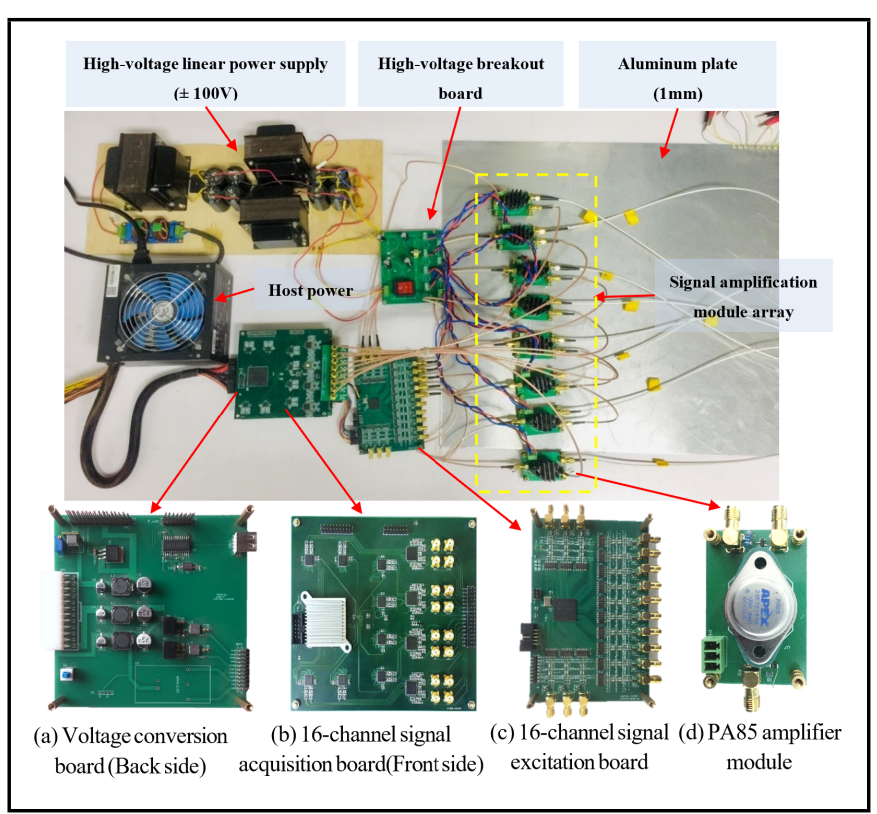

Figure 10. System connection diagram.

Table 3. Delay time for each channel.

\begin{tabular}{|c|c|c|c|c|c|c|c|c|}
\hline Channel & CH1 & CH2 & CH3 & CH4 & CH5 & CH6 & CH7 & CH8 \\
\hline Delay time (ns) & 9970 & 8570 & 7160 & 5745 & 4320 & 2885 & 1445 & 0 \\
\hline
\end{tabular}

The experimental setup is shown in Fig. 10. Firstly, only excitation and receiving in channel 1 was implemented. Then, beam focusing was performed at the defect position, also in the non-defective area. The delay time of each channel was determined by the distance difference between the defect position and each element of the sensor array. All the calculated delay excitation time required is shown in Table 3. Finally, the guided wave (only $S_{0}$ mode) phased focusing was realized at the defect position. The results are shown in Fig. 11.

It can be seen from the results that there was no defect echo, excitation and receiving only by $\mathrm{CH} 1$. Then, if each channel transmits with a preset delay time, the 8-channel superimposed signal obtained after focusing on the defect position, we can clearly observe the defect echo. It is shown that the acoustic beam can be focused by phased deflection, so that the guided wave energy will be able to be focused on the defect. Then, the most area of the specimen can be examined by electronical scanning. It greatly increases the amplitude of the defect echo, and effectively improves the detection rate and detection area of small defects. It also proves that the system can realize multi-channel guided wave phased focusing detection based on

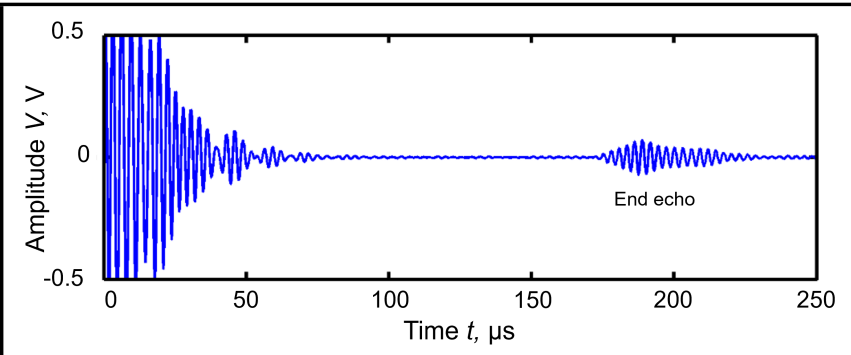

(a) Excitation and receiving only by $\mathrm{CH} 1$

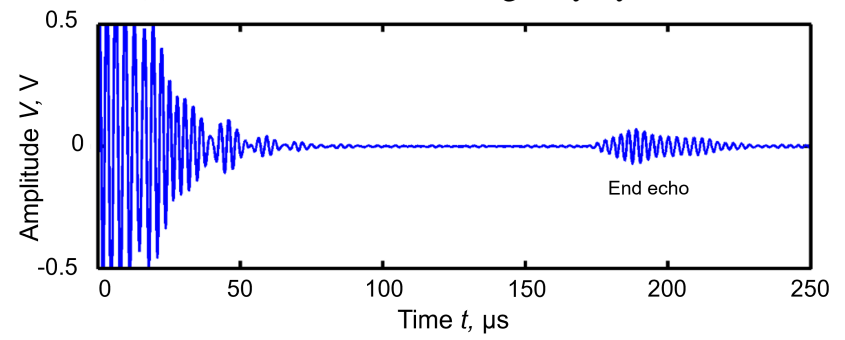

(b) Phased array focusing signal

Figure 11. Phased array focusing detection results.

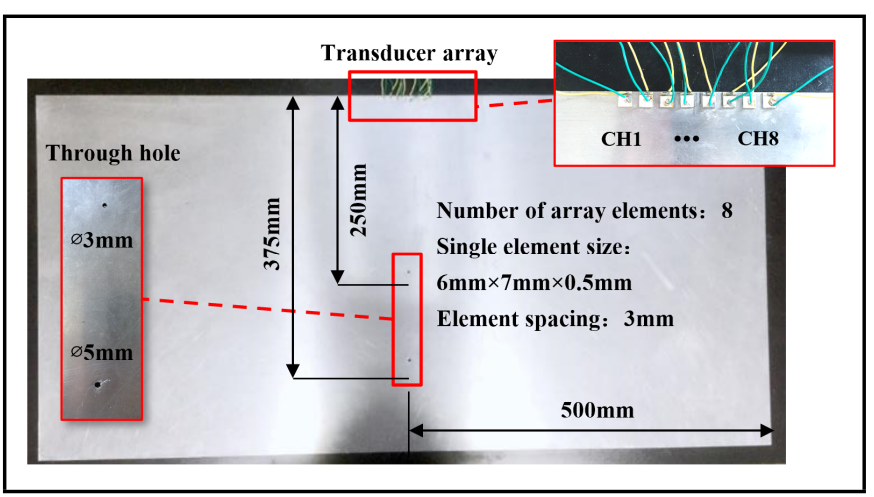

Figure 12. Total focusing experiment.

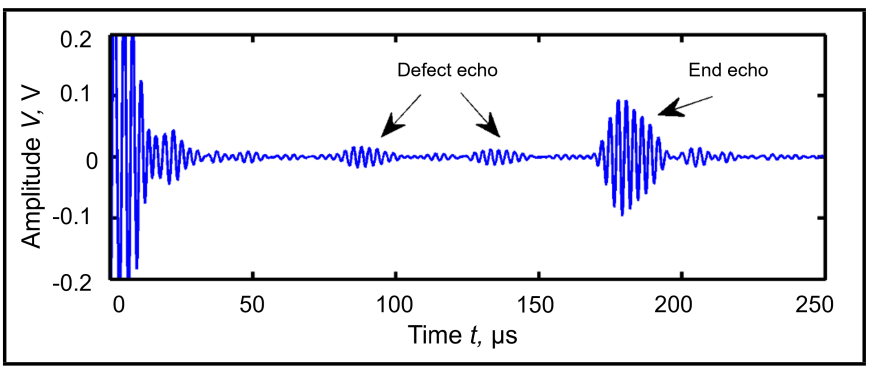

Figure 13. Time domain waveform (Excitation and receiving by $\mathrm{CH} 4$ )

sensor array.

\subsection{Thin Plate TFM Imaging}

The developed multi-channel guided wave detection system was also used to conduct total focusing imaging experiments on the same aluminum thin plate. However, multiple defects were employed, and their locations and sizes as shown in Fig. 12.

From the typical time-domain waveform (only using $\mathrm{CH} 4$ ) obtained from the experimental system as shown in Fig. 13, two defect echo signals can be observed. Base on the total focusing algorithm, guided-wave total focusing imaging was performed using the full-matrix data. The imaging resolution was set to $1 \mathrm{~mm}$. To eliminate the artifacts formed by the noise, 


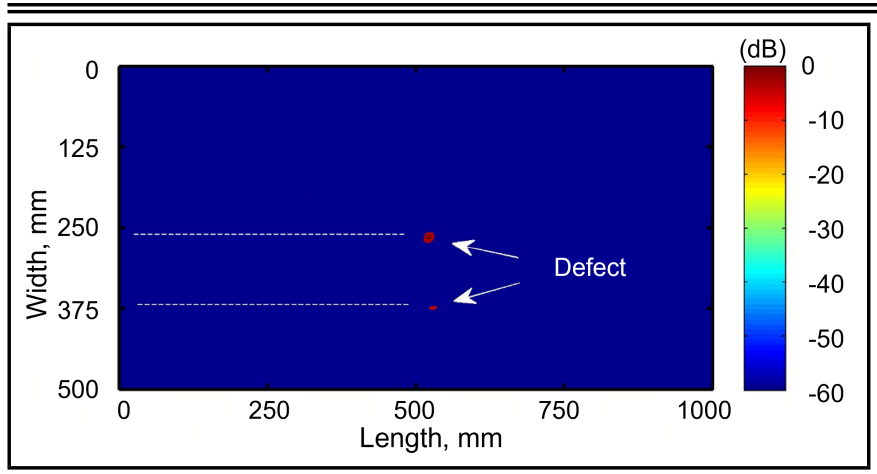

Figure 14. Guided wave total focusing imaging results.

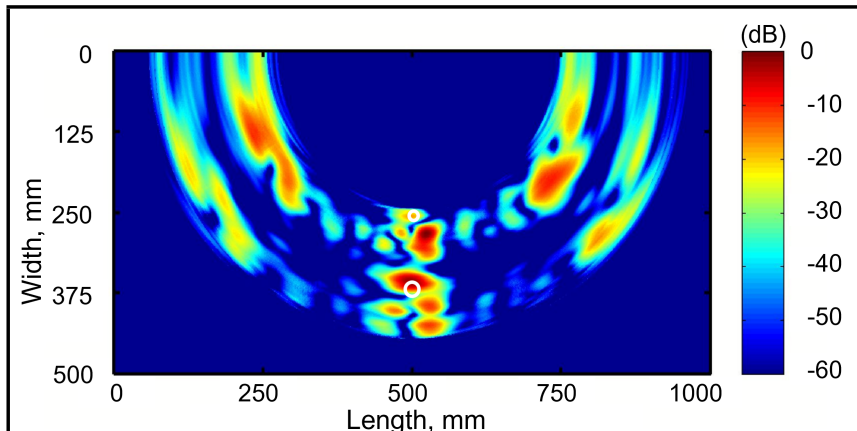

(a) TFM

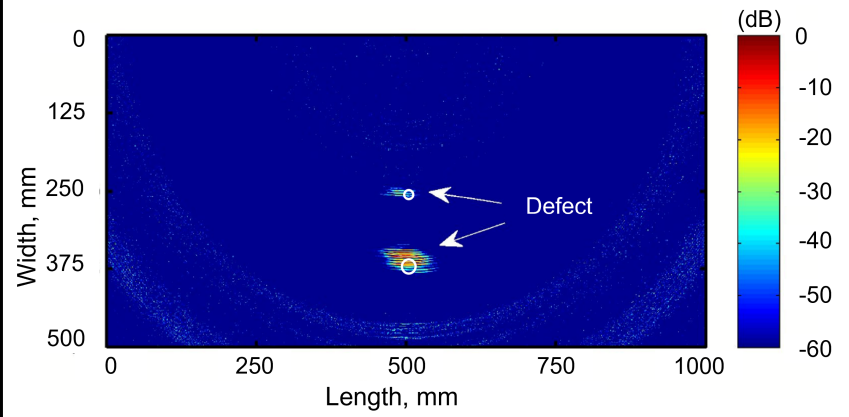

(b) $\mathrm{SCF}$

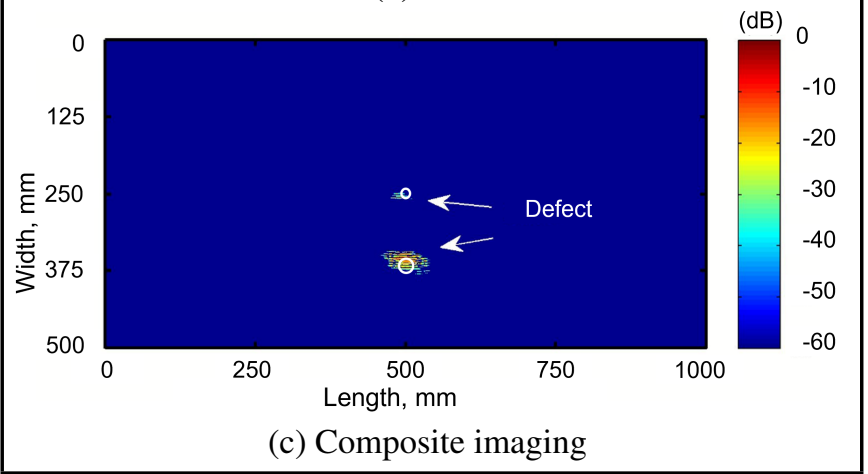

Figure 15. Imaging results (Actual defect positions and sizes are indicated in white circles).

the imaging result was subjected to a threshold of $90 \%$, as shown in Fig. 14.

According to the results of the threshold total focusing imaging, two defects were detected, and their accurate position can be obtained. Compared with the previous point-by-point experimental data collection, the efficiency is significantly improved through the automatically electronical scanning.

\subsection{Guided Wave Composite Imaging}

Using the full matrix data obtained in the TFM imaging experiment, three images were obtained in total. These images were the amplitude TFM image, SCF image, and the composite total focusing image. All the results were without threshold. All the imaging results are shown in Fig. 15.

There were many virtual artifacts caused by noise in amplitude TFM imaging without threshold processing, as shown in Fig. 15(a), in which it was difficult to accurately determine the defects' location. SCF imaging eliminated most of the artifacts, and it can accurately identify the location of the two defects. However, there are still some artifacts, as shown in Fig. 15(b). Combined with the advantages of high contrast of amplitude imaging and high resolution of phase imaging, the fusion of these two methods eliminated all the artifacts while keeping the defects located.

However, the TFM imaging mainly uses defect scattering information in a regular reflection area, and the full matrix data collected by the linear sensor array can only receive part of the scattering information. It is difficult to obtain the scattering information in the whole area, so the defect size and its morphological characteristics cannot be accurately determined.

\section{CONCLUSIONS}

In this research, a 16-channel guided wave detection system is developed to achieve the guided wave array detection. It can generate sinusoidal tone-burst waveform signals, and the amplitude of the excitation signal can reach $150 \mathrm{Vpp}$. The resolution of the excitation delay time can reach $5 \mathrm{~ns}$. The 16channel synchronous acquisition module has a sampling rate of $50 \mathrm{MS} / \mathrm{s}$. It realizes the automatic acquisition and storage of full matrix data, which avoids the tedious and inefficient manually experiments of guided wave array detection by distributed sensors.

Based on this system, defect detection is carried out on a thin aluminum plate. The phased focusing detection and the total focusing imaging of defects have been applied, which proves the practicability and reliability of the system. The fusion of amplitude TFM imaging and phase based SCF imaging is also implemented. The composite imaging results can eliminate the noise artifacts and improve the imaging contrast. All the demonstrated experiments and imaging methods set a guideline for multi-transducer guided wave detection. In this paper, the linear sensor array can only receive partial defect scattering information. Thus, it cannot obtain the size and morphological characteristics of the defect. But the developed system is still flexible for the distributed sensor array detection, such as phased focused scanning imaging, ultrasonic guided waves computerized tomography, ${ }^{23}$ and positioning imaging methods based on the propagation time of defective wave packets (elliptical imaging algorithms, hyperbolic imaging algorithms, etc. $\left.{ }^{24,25}\right)$.

\section{ACKNOWLEDGMENTS}

This work is supported by national Natural Science Foundation of China (Nos. 11872082, 51875010), and Beijing Natural Science Foundation (No. 3192006)

\section{REFERENCES}

1 Rose, J. L. A baseline and vision of ultrasonic guided wave inspection potential, JOURNAL OF PRESSURE VESSEL 
TECHNOLOGY-TRANSACTIONS OF THE ASME, 124 (3), 273-282, (2002). https://dx.doi.org/10.1115/1.1491272

2 Cho, Younho., Hongerholt, D. D. , and Rose, J. L.Lamb wave scattering analysis for reflector characterization, IEEE Transactions on Ultrasonics Ferroelectrics \& Frequency Control, 44 (1),44-52, (1997). https://dx.doi.org/10.1109/58.585189

3 Zhao, X. , Royer, R. L. , Owens, S. E. , and Rose, J. L. Ultrasonic lamb wave tomography in structural health monitoring, Smart Materials \& Structures, 20 (10), 105002, (2011). https://dx.doi.org/10.1088/0964$1726 / 20 / 10 / 105002$

${ }^{4}$ Huang, S., Wei, Z. , Zhao, W. , and Wang, S. A new omni-directional emat for ultrasonic lamb wave tomography imaging of metallic plate defects, Sensors, 14 (2), 3458-3476, (2014). https://dx.doi.org/10.3390/s140203458

5 Drinkwater, B. W. , and Wilcox, P. D. Ultrasonic arrays for non-destructive evaluation: a review, NDT\&E International, 39 (7), 525-541, (2006). https://dx.doi.org/10.1016/j.ndteint.2006.03.006

6 Michaels, J. E. , and Michaels, T. E. Enhanced differential methods for guided wave phased array imaging using spatially distributed piezoelectric transducers, Annual Review of Process in Quantitative Nondestructive Evaluation, (2006). https://dx.doi.org/10.1063/1.2184613

7 Wilcox, P. D., Holmes, C. , and Drinkwater, B. W. Advanced reflector characterization with ultrasonic phased arrays in nde applications, IEEE Transactions on Ultrasonics Ferroelectrics and Frequency Control, 54 (8), 1541-1550, (2007). https://dx.doi.org/10.1109/TUFFC.2007.424

8 Zhang, J., Drinkwater, B. W., and Wilcox, P. D. The use of ultrasonic arrays to characterize crack-like defects, Journal of Nondestructive Evaluation, 29 (4), 222-232, (2010). https://dx.doi.org/10.1007/s10921-010-0080-6

9 Meola, C. , Boccardi, S. , Carlomagno, G. M. , Boffa, N. D. , Monaco, E. , and Ricci, F. Nondestructive evaluation of carbon fibre reinforced composites with infrared thermography and ultrasonics, Composite Structures, 134 (DEC.), 845-853, (2015). https://dx.doi.org/10.1016/j.compstruct.2015.08.119

10 Long, R. , Russell, J. , and Cawley, P. Ultrasonic phased array inspection using full matrix capture, Insight, 54 (7), 380-385, (2012). https://dx.doi.org/10.1784/insi.2012.54.7.380

11 Caminero, M. A., Garcia-Moreno, I. , Rodriguez, G. P. , and Chacon, J. M. Internal damage evaluation of composite structures using phased array ultrasonic technique: impact damage assessment in cfrp and $3 \mathrm{~d}$ printed reinforced composites, Composites, 165 (MAY15), 131-142, (2019). https://dx.doi.org/10.1016/j.compositesb.2018.11.091

12 Clay, A. C. , Wooh, S. C., Azar, L., and Wang, J. Y. Experimental study of phased array beam steering characteristics, Journal of Nondestructive Evaluation, 18 (2), 59-71, (1999). https://dx.doi.org/10.1023/A:1022618321612

$13 \mathrm{Hu}$, C. H. , Zhang, L. Q. , Cannata, J. M. , Yen, J. , and Shung, K. K. Development of a 64 channel ultrasonic high frequency linear array imaging system, Ultrasonics, 51 (8), 953-959, (2011). https://dx.doi.org/10.1016/j.ultras.2011.05.010

14 Li, J. , and Rose, J. L. Angular-profile tuning of guided waves in hollow cylinders using a circumferential phased array, IEEE Transactions on Ultrasonics, Ferroelectrics and Frequency Control, 49 (12), 1720-1729, (2002). https://dx.doi.org/10.1109/TUFFC.2002.1159849

15 Holmes, C. , Drinkwater, B. W. , and Wilcox, P. D. Post-processing of the full matrix of ultrasonic transmit-receive array data for non-destructive evaluation, NDT\&E International, 38 (8), 701-711, (2005). https://dx.doi.org/10.1016/j.ndteint.2005.04.002

16 Jeune, L., Robert, S. , Dumas, P. , Membre, A. , and Prada, C. Adaptive ultrasonic imaging with the total focusing method for inspection of complex components immersed in water, Annual Review of Progress in Quantitative Nondestructive Evaluation, 1650 (1), 1037-1046, (2015). https://dx.doi.org/10.1063/1.4914712

17 Li, C. , Pain, D. , Wilcox, P. D. , and Drinkwater, B. W. Imaging composite material using ultrasonic arrays, NDT\&E International, 53 (16): 8-17, (2013). https://dx.doi.org/10.1016/j.ndteint.2012.07.006

18 Prado, V. T. , Higuti, R. T. , Kitano, Cláudio, MartínezGraullera, óscar, and Adamowski, J. C. Lamb mode diversity imaging for non-destructive testing of plate-like structures, NDT\&E International, 59 (7), 86-95, (2013). https://dx.doi.org/10.1016/j.ndteint.2013.06.001

19 O’Donnell, M. Phase-insensitive pulse-echo imaging, Ultrasonic Imaging, 4 (4), 321-335, (1982). https://dx.doi.org/10.1016/0161-7346(82)90016-5

${ }^{20}$ Liu, Z. H. , Sun, K. M. , and Song, G. R. Damage localization in aluminum plate with compact rectangular phased piezoelectric transducer array, Mechanical Systems and Signal Processing, 70-71, 625-636, (2016). https://dx.doi.org/10.1016/j.ymssp.2015.09.022

21 Camacho, J. , Parrilla, M. , and Fritsch, C. Phase coherence imaging, IEEE transactions on ultrasonics, ferroelectrics, and frequency control, 56 (5), 958-974, (2009). https://dx.doi.org/10.1109/TUFFC.2009.1128

${ }^{22}$ Higuti, R. T. , Martinez-Graullera, O. , Carlos J Martín, Octavio, A. , and Espinosa, F. M. D. Damage characterization using guided-wave linear arrays and image compounding techniques, IEEE Transactions on Ultrasonics Ferroelectrics and Frequency Control, 57 (9), 1985-1995, (2010). https://dx.doi.org/10.1109/TUFFC.2010.1646

${ }^{23}$ Leonard, K. R., Malyarenko, E. V. , and Hinders, M. K. Ultrasonic lamb wave tomography, Inverse Problems, $\mathbf{1 8}$ (6), 1795-1808, (2002). https://dx.doi.org/10.1088/0266$5611 / 18 / 6 / 322$

${ }^{24}$ Crawley, E. F. , and De Luis, J. Use of piezoelectric actuators as elements of intelligent structures, AIAA Journal, 25 (10), 1373-1385, (1987). https://dx.doi.org/10.2514/3.9792

25 Clarke, T. , Simonetti, F., and Cawley, P. Guided wave health monitoring of complex structures by sparse array systems: influence of temperature changes on performance, Journal of Sound \& Vibration, 329 (12), 2306-2322, (2010). https://dx.doi.org/10.1016/j.jsv.2009.01.052 